BNL-107196-2014-JA

\title{
Photomultiplier tube failure under hydrostatic pressure in future neutrino detectors
}

\author{
K. Chambliss, ${ }^{a}$ S.K. Sundaram, ${ }^{a, 1}$ N. Simos ${ }^{b}$ and M. Diwan ${ }^{b}$ \\ ${ }^{a}$ Multifunctional Materials Laboratory (M\&M Lab), Kazuo Inamori School of Engineering, \\ Alfred University, Alfred, NY 14802, U.S.A. \\ ${ }^{b}$ Brookhaven National Laboratory, \\ Upton, NY 11973, U.S.A. \\ E-mail: sundaram@alfred.edu
}

\begin{abstract}
Failure of photomultiplier tubes (PMTs) under hydrostatic pressure is a concern in neutrino detection, specifically, in the proposed Long-Baseline Neutrino Experiment project. Controlled hydrostatic implosion tests were performed on prototypic PMT bulbs of 10-inch diameter and recorded using high speed filming techniques to capture failures in detail. These high-speed videos were analyzed frame-by-frame in order to identify the origin of a crack, measure the progression of individual crack along the surface of the bulb as it propagates through the glass, and estimate crack velocity. Crack velocity was calculated for each individual crack, and an average velocity was determined for all measurable cracks on each bulb. Overall, 32 cracks were measured in 9 different bulbs tested. Finite element modeling (FEM) of crack formation and growth in prototypic PMT shows stress concentration near the middle section of the PMT bulbs that correlates well with our crack velocity measurements in that section. The FEM model predicts a crack velocity value that is close to the terminal crack velocity reported. Our measurements also reveal significantly reduced crack velocities compared to terminal crack velocities measured in glasses using fracture mechanics testing and reported in literature.
\end{abstract}

KEYWORDS: Cherenkov detectors; Materials for solid-state detectors; Photon detectors for UV, visible and IR photons (vacuum) (photomultipliers, HPDs, others); Neutrino detectors

\footnotetext{
${ }^{1}$ Corresponding author.
} 


\section{Contents}

1 Introduction 1

1.1 Background 1

2 Materials and methods $\quad 2$

2.1 Crack velocity measurement 2

2.2 3-D modeling and curvature corrections 3

2.3 Finite element modeling (FEM) of crack initiation and propagation 4

3 Results and discussion $\quad 5$

4 Conclusion 9

\section{Introduction}

Photomultiplier tube (PMT) is a component that is critical to successful neutrino detection experiments such as the Super-Kamiokande (Super-K) detector[1], the Sudbury Neutrino Observatory (SNO)[2], and the water Cherenkov detector option for the Long-Baseline Neutrino Experiment (LBNE)[3]. The PMTs are exposed to large hydrostatic pressures as well as prolonged contact with high purity water during these experiments. The detector proposed for the LBNE would house PMTs at depths of up to $80 \mathrm{~m}$, exposing them to external hydrostatic pressures of approximately 129 psi[4]. Similarly, for the Hyper-Kamiokande Experiment[5], the PMTs will be under a hydrostatic pressure of about 68 psi of ultra-pure water. Sustained PMT performance over the proposed approximate 20 years lifetime is required to protect the light-sensitive devices. The water purification system is needed for efficient collection of Cherenkov light over long distances; it will be designed based on previous experience, where cycling ultra-pure water ranged in resistivity from 11 to $18.24 \mathrm{M} \Omega-\mathrm{cm}^{1}$. Despite long experience, the effects of this environment on stability and strength of PMT glass are not well known and could contribute to failure.

\subsection{Background}

One primary issue facing the long-term success of these experiments is structural failure of the PMTs, which could potentially lead to catastrophic cascade failures in the large PMT arrays, especially when the shockwave from a single PMT bulb failure is intense enough to trigger failure in neighboring PMT bulbs. Such failures can disrupt and risk of disrupting or even ending long-term experiments. Individual bulb failures can result from a number of different factors affecting performance, including bulb shape, thickness of the glass, surface or bulk flaws, stress corrosion, residual stresses from processing, and extended exposure to the environmental conditions of neutrino detection, such as ultra-pure water, hydrostatic pressure, extended exposure up to twenty years. The 
crack velocity and subsequent force generated in a failing PMT bulb shape the intensity of the resulting shockwave, making it an important characteristic to understand regarding these failures[6]. Our ultimate goal is to analyze both experimental and simulation crack propagation data and draw critical parameters (such as stress intensity and/or crack initiation information from critical size microcracks in these type of glass) that will help determine the likelihood of failure under the anticipated conditions surrounding these PMTs and their life expectancy. In this report, we present our early result obtained towards that goal.

BNL has conducted prototypic tests concerning the reliability of PMTs under hydrostatic pressure at the Naval Undersea Warfare Center (NUWC) in Newport, Rhode Island. In these tests, PMTs were secured near the center of a $15 \mathrm{~m}$ diameter, spherical steel tank, and submerged in filtered water. The tank was pressurized using an air pump up to 88 psi at the center of the vessel and failure was induced by impact of a steel bolt plunger. The primary objective of these tests was to observe and measure the pressure wave following a PMT bulb failure. Once fracture initiated, the complete obliteration of the bulb lasted only a few milliseconds, as water filled the volume inside the bulb, which was previously under vacuum. The inward rush of water stopped upon impact of the water on itself, thus creating a shockwave moving outward[6]. This study[7] on the survival of an assembled array of PMTs under significant hydrostatic pressure and subjected to shock waves caused by the failure of a single PMT has shown that careful design can effectively reduce the magnitude of the shock wave in the future experiments[8].

\section{Materials and methods}

\subsection{Crack velocity measurement}

Following the shockwave tests at the NUWC, a series of single implosion tests in a small pressure vessel were conducted at BNL with the primary objective of inducing and observing in detail the failure of PMT bulbs under hydrostatic pressure. A variety of PMT bulbs were obtained from manufacturers. The results reported here were based on test on the Hamamatsu R7081 PMT bulb, which had a nominal diameter of $25 \mathrm{~cm}$, however the detailed shape of the bulb was used in our analysis. The testing procedure was such that PMT specimens would be subjected to a pressure rise from zero to 300 psi over a time span of five minutes, two hours, or 24 hours. The failure was induced by pressurization alone and het by a steel bolt. Pressurization was controlled using National Instruments' LabVIEW software to ensure consistent rates of loading and to record the pressure at failure of each PMT. Each test and failure, if it occurred, was recorded using a highspeed camera, filming at speeds of 4,000-6,000 frames per second, in order to capture the mode and progression of failure in each specimen in detail. These high-speed recordings allowed us to follow the growth of cracks in the bulb surface and measure crack velocity in real world application. We present our experimental and modeling results on crack velocity measurements and validation.

Analysis of high-speed videos taken at BNL began with the identification of failure origins and resolution of measurable cracks. Each test recording was examined frame-by-frame in order to measure the propagation of individual cracks along the surface of the sample. Measured cracks were selected based on visibility and traceable propagation through several frames of video. Once selected, identifying the crack tip in the earliest possible frame on screen and marking the spot with 


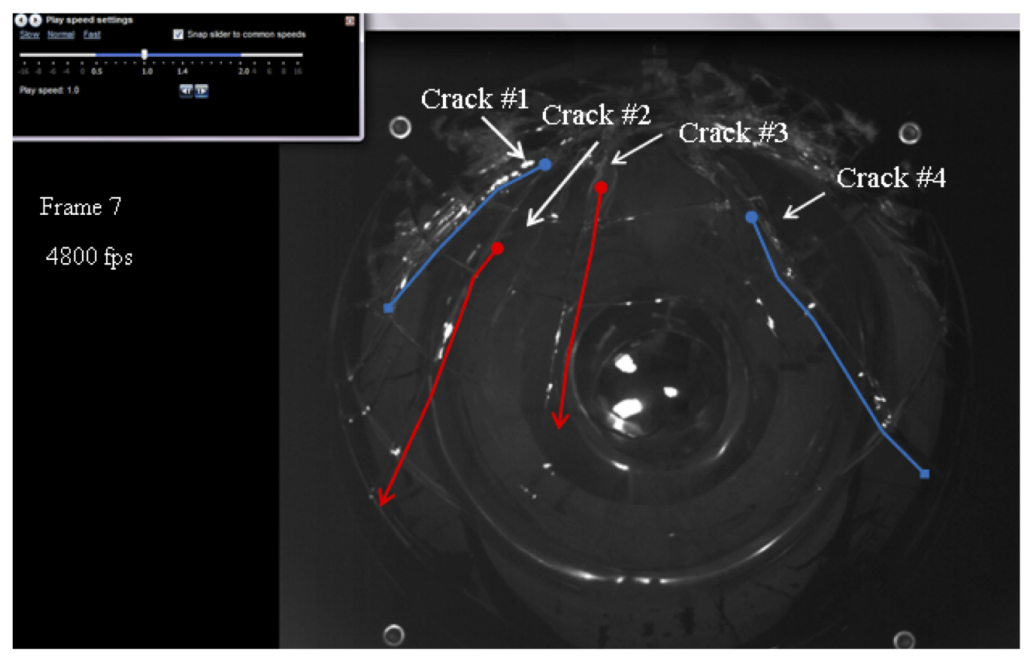

Figure 1. Example of a single frame used for crack measurement. The bulb is being viewed from the top down. Red arrow: cracks being measured and Blue arrow: measurements completed.

masking tape measured the propagation distances. The video was first advanced by a single frame, the new position of the crack tip was marked, and then the distance from the original mark was then recorded. This procedure was repeated throughout all measurable frames to obtain a total distance of crack propagation (figure 1). Scaling factors were determined for each individual video using the known diameter of the bulbs (10 inches) as compared to the measured, on-screen diameter. Crack velocity was calculated for individual cracks and an average velocity was found for all measurable cracks on each specimen using the scaled propagation distances and time values obtained from the known frame rate. For example, in the case of a crack of total length of $9.2 \mathrm{~cm}$ measured at a frame rate of $4000 \mathrm{fps} \rightarrow 3$ frames $=3 / 4000 \mathrm{sec}:$

$$
\text { Average Velocity }=(\text { Total } \Delta \text { Length }) / \text { Time }=9.2 \mathrm{~cm} /(3 / 4000 \mathrm{sec})=123 \mathrm{~m} / \mathrm{sec}
$$

The curvature of the bulb increases the actual crack length relative to the measured length; therefore the actual crack velocity needed further corrections with a detailed model of the bulb shape.

\subsection{3-D modeling and curvature corrections}

In order to correct the initial crack velocities, a 3-D computer model of a 10-inch Hamamatsu PMT bulb, created at BNL, was used as a projection surface for the crack distances measured from the implosion recordings. The crack paths from two-dimensional video measurements were transferred into Dassault Systèmes' SolidWorks modeling software. The crack tip positions had to be measured in each frame of video, using the center peak of the bulb as a reference point. By noting the distance from the bulb center and an angle from vertical, the crack tip positions could be accurately transferred into the bulb model. These positions were sketched into the computer-aided design (CAD) drawing in the form of rays radiating from the reference point on a plane perpendicular to the bulb peak (figure 2). Connecting the outer ends of the rays gave a representation of the total crack path, which was then projected onto the surface of the model, giving curvature of the crack itself (figure 3). The arc lengths of the new, curved paths were measured using the built-in 


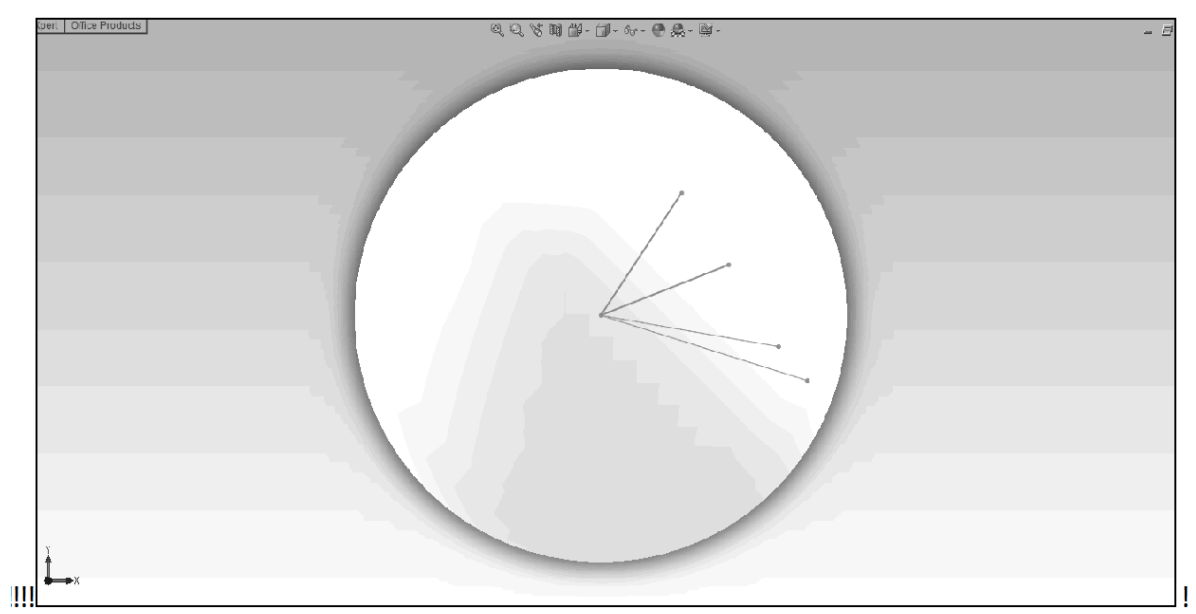

Figure 2. A top-down view of the bulb model; each line represents a measurement point along the crack path, relative to the central peak of the bulb.

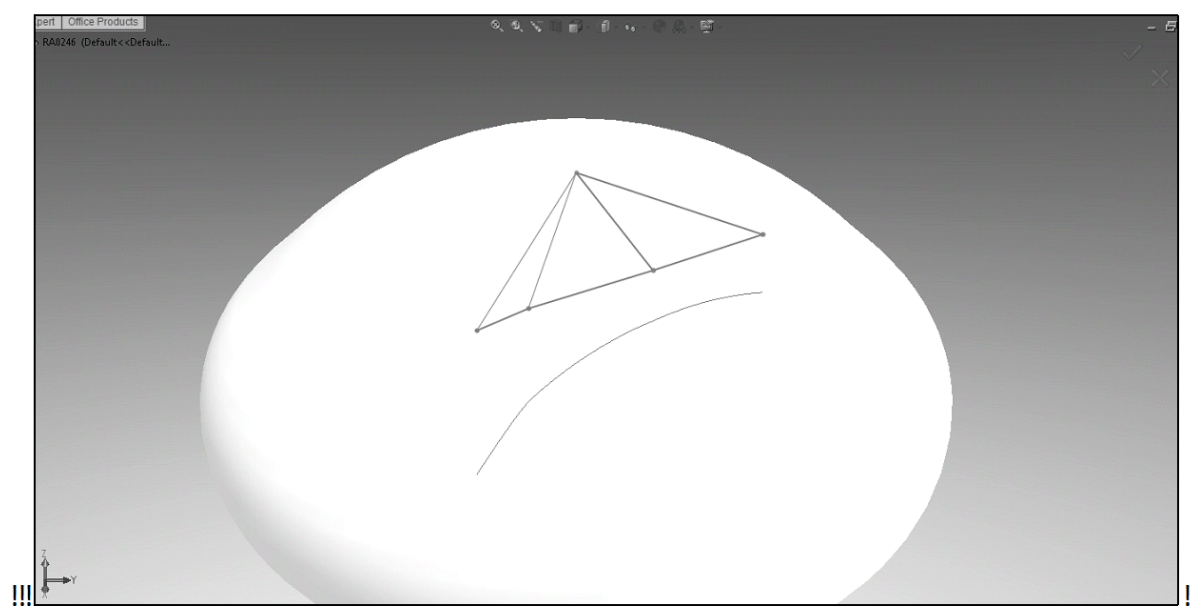

Figure 3. In a rotated view of the bulb model, the segments from figure 2 are visible, in dark gray, connected at their ends by the 2-D representation of the crack path. The light gray arc represents the intersection of the projection of the 2-D crack path and the surface of the bulb model.

measure tool in SolidWorks. The arc lengths were used to calculate corrected crack velocities for each individual crack, as well as averages for each PMT, and an overall average for all samples.

\subsection{Finite element modeling (FEM) of crack initiation and propagation}

In an effort to assess the mechanisms leading to PMT failure and identify the external pressure thresholds of the glass structure three numerical approaches were considered. A non-linear plasticity damage model, capable of describing damage in ceramics, glass and other brittle materials was utilized in analyses based on LS-DYNA[9] non-linear finite element code. Specifically, the PMT glass constitutive and damage behavior was first assumed to follow the Johnson-Holmquist ceramic model, which is included in the LS-DYNA materials library and is useful for modeling ceramics, glass and other brittle materials and incorporates into its damage accumulation process, 
Table 1. Glass properties used for the FEM.

\begin{tabular}{|l|c|c|}
\hline Properties & Baseline Analysis & Sensitivity Analysis \\
\hline Density $\left(\mathrm{Kg} / \mathrm{mm}^{3}\right)$ & $2.23 \times 10^{-6}$ & $2.23 \times 10^{-6}$ \\
\hline Elastic modulus $(\mathrm{psi})$ & 9282415 & 9282415 \\
\hline Poisson's ratio & 0.42 & 0.42 \\
\hline Ultimate compressive strength $(\mathrm{psi})$ & 58 & 58 \\
\hline Ultimate tensile strength $(\mathrm{psi})$ & 5076 & 5076 \\
\hline Fracture energy $\left(\mathrm{J} / \mathrm{m}^{2}\right)$ & 8 & $3-16$ \\
\hline
\end{tabular}

amongst other key properties, glass fractured strength strain-rate strength, Hugoniot elastic limit as well as elastic energy loss converting to hydrostatic energy that in turn affects instability triggering pressures. This complex plasticity damage model, however, predicted that the PMT is capable of sustaining $\sim 3521$ psi of hydrostatic pressure before instability and collapse is initiated, which far exceeds the observed limiting pressures during hydrostatic failure tests performed at BNL. Anticipating that the triggering of failure in the PMT is not glass strength dependent but rather instability failure (i.e. instabilities induced by the external pressure in the form of buckling in the PMT structure), an instability analysis was carried out that revealed instability modes at $\sim 294$ psi of pressure which is close to the experimentally observed failure pressures. Subsequently, in order to understand the crack formation in the prototypic PMT, the Winfrith concrete model[10] was adopted by introducing the PMT glass properties into the constitutive model including fracture energies for crack formation. This material model is part of the material library of LS-DYNA[11] and is a plasticity model, which has cracking capabilities and includes the third stress invariant for consistently treating both triaxial compression and triaxial extension In this model, the strain-rate effects and the fracture energy were included in addition to the elastic constants of the glass.

Table 1 shows the properties used in the baseline analysis where the fracture energy was assumed to be $8 \mathrm{~J} / \mathrm{m}^{2}$. Sensitivity studies around the assumed fracture energy (as low as $3 \mathrm{~J} / \mathrm{m}^{2}$ and as high as $16 \mathrm{~J} / \mathrm{m}^{2}$ ) showed very small variation in the initiation of the cracking. The finite element model used in the PMT damage analysis consisted of $\sim 216,000$ three dimensional glass elements with five elements across the PMT thickness. The finite element model that represented the geometrical shape of the PMT precisely was generated using the capabilities of the TrueGrid[12] code. A pressure time history was applied to the entire external surface of the PMT model. The total duration of the simulated pressure history was of the order of $1 \mathrm{~ms}$, which was dictated by the computational demand of the analysis. In future analyses however, the actual pressure history of the BNL PMT hydrostatic tests will be adopted to assess the effect of damage accumulation. The PMT deformation, stress concentrations, crack formation, and crack evolution were tracked as a function of increased hydrostatic pressure.

\section{Results and discussion}

In all, 32 cracks were measured between nine separate implosion tests and an overall average velocity was calculated. A summary of the results measured for each bulb is given in table 2 including standard deviation and pressure at failure. With the initial method, the average velocity 
Table 2. Crack velocity data summary.

\begin{tabular}{|c|c|c|c|c|c|c|c|}
\hline $\begin{array}{c}\text { Bulb } \\
\text { Designation }\end{array}$ & $\begin{array}{l}\text { Pressure } \\
\text { at Break } \\
\text { (psi) }\end{array}$ & $\begin{array}{c}\text { Time } \\
\text { Span Used }\end{array}$ & $\begin{array}{l}\text { \# of Cracks } \\
\text { Measured }\end{array}$ & $\begin{array}{c}\text { Average } \\
\text { Crack Velocity } \\
\text { with Initial Method } \\
(\mathrm{m} / \mathrm{sec})\end{array}$ & $\begin{array}{l}\text { Standard } \\
\text { Deviation } \\
(\mathrm{m} / \mathrm{sec})\end{array}$ & $\begin{array}{c}\text { Average } \\
\text { Crack Velocity } \\
\text { with Curve Correction } \\
(\mathrm{m} / \mathrm{sec})\end{array}$ & $\begin{array}{c}\text { Standard } \\
\text { Deviation } \\
(\mathrm{m} / \mathrm{sec})\end{array}$ \\
\hline RA0246 & 260 & 5 min, induced & 4 & 192 & 64 & 202 & 71 \\
\hline RA0227 & 269 & 5 min, induced & 1 & 192 & - & 201 & - \\
\hline TA3610 & 205 & 5 min, self-implosion & 3 & 239 & 57 & 251 & 107 \\
\hline TA3281 & 103 & 5 min, induced & 5 & 144 & 37 & 160 & 45 \\
\hline TA3522 & 294 & $2 \mathrm{hr}$, self & 4 & 250 & 132 & 313 & 185 \\
\hline TA3598 & 299 & $2 \mathrm{hr}$, self & 6 & 149 & 30 & 175 & 33 \\
\hline TA3624 & 130 & $24 \mathrm{hr}$, self & 3 & 168 & 15 & 192 & 24 \\
\hline TA3410 & 132 & $2 \mathrm{hr}$, self & 3 & 242 & 110 & 276 & 123 \\
\hline TA3479 & 215 & $2 \mathrm{hr}$, self & 3 & 275 & 102 & 317 & 132 \\
\hline
\end{tabular}

was found to be $206 \mathrm{~m} / \mathrm{s}$ with a standard deviation of $47 \mathrm{~m} / \mathrm{s}$. When the curvature correction procedure was applied, the average velocity was $232 \mathrm{~m} / \mathrm{s}$ with a standard deviation of $59 \mathrm{~m} / \mathrm{s}$. The curve correction procedure shows a $13 \%$ increase in the average measured velocity and a $24 \%$ increase in standard deviation. We have further analysed the systematic errors on the our measurements as follows: the resolution for all our data was at least 512 pixels by 512 pixels spanning a view of $30 \mathrm{~cm} \times 30 \mathrm{~cm}$ at the dome of the PMT bulb. Based on trial and error, the estimate for identifying the crack tip and end is $<3 \mathrm{~mm}$. Given the average length of a measured crack of $\sim 10 \mathrm{~cm}$, the error from crack reconstruction is $<5 \%$. The reconstructed crack had to be corrected for the shape of the bulb as described above. We use the Hamamatsu tolerance specification[13] for the $25 \mathrm{~cm}$ bulb dimension of $\pm 5 \mathrm{~mm}$ to estimate that our correction adds $<5$ $\%$ error. The error from the specification of the frame rate of the camera was negligible. Finally, the largest error comes from the limited number of frames - typically 3 frames - that we used to measure the time of crack propagation. Since we cannot determine if the crack stopped at the beginning or the end of a frame, we assign an error of $\pm 1 / 2$ frame to this measurement. Adding the above contributions in quadrature, we estimate that the total systematic error on our measurement of crack velocity is less than $20 \%$.Generally, the average velocity values measured are higher at locations close to the middle section of the PMT bulbs, where the diameter is the largest compared to the values close to the top of the bulbs. As it is difficult to measure instantaneous crack velocity in situ, the average velocity remains the most representative of these measurements. Additionally, glass fracture is obviously statistical in nature due to inherent flaws present and their population, which depends on the thermal history and glass composition. The standard deviation, therefore, could be considered representative of the variations in the material.

The analysis based on the glass cracking FEM model and the constitutive relations of the Winfrith Concrete model were used to assess both initiation of failure, crack formation and tracing or evolution as a result of increased hydrostatic pressure on the PMT outer surface. The analysis proceeded with incremental pressure, while the tri-axial stress conditions within the glass material along with the damage criteria and the assumed fracture energies of the PMT glass determined the formation and the evolution of cracking. figures 4(a) and 4(b) show FEM results of crack initiation and progression in prototypic PMTs. 

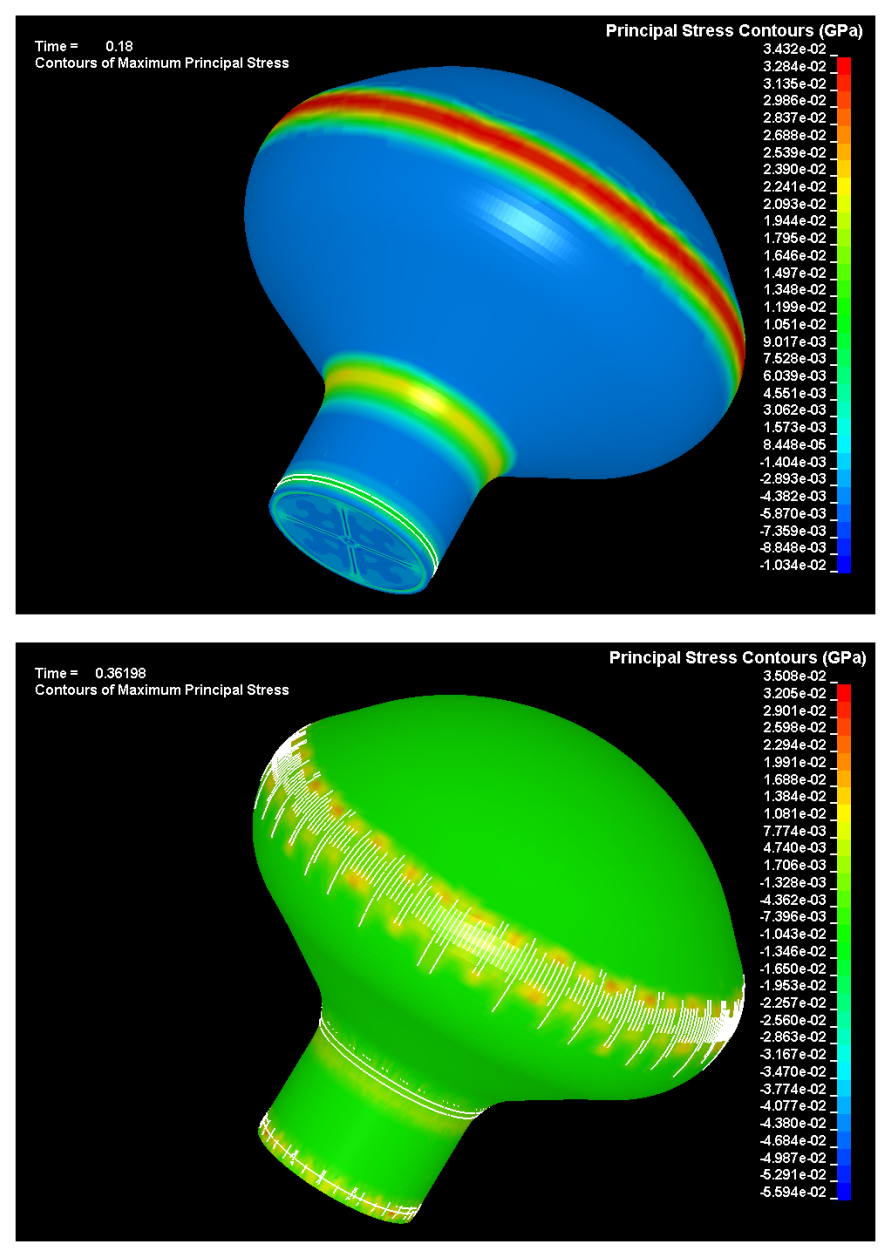

Figure 4. Crack Initiation at the base and progression in the PMT at: (a) $t=0.18 \mathrm{~ms}$ and (b) $t=0.36 \mathrm{~ms}$ (higher resolution).

The analysis predicts that crack initiation occurs at the cylindrical base of the PMT first, followed by the middle (at largest diameter) section and subsequently by the transition (lower) section. The pressure at the initiation of cracking is around $350 \mathrm{psi}$, which is reasonably close to the value observed in the prototypic testing in BNL.

Figure 5(a) and 5(b) show the FEM results of crack initiation and propagation selected on the inside of the PMT. The coordinates of the points in the vicinity of the cracks in question are in $\mathrm{mm}(\mathrm{x}, \mathrm{y}, \mathrm{z})$. Thus, by observing the movement of the crack and the length that it extends between times (shown on figure 5(b)), the crack velocity can be measured. Higher stress concentrations were observed around the middle section of the PMT bulb with highest diameter, suggesting that section was weak. Our experimental crack velocities in this section measured higher compared to other sections, supporting our observation from the FEM results.

Our data does not show any direct correlation between the external pressure of testing and the crack velocity. Table 2 shows details of the pressures of testing used for different PMTs. The crack velocities measured using this video recording method are well below reported terminal crack velocity of reference glasses measured using fracture mechanics testing methods. For examples, 

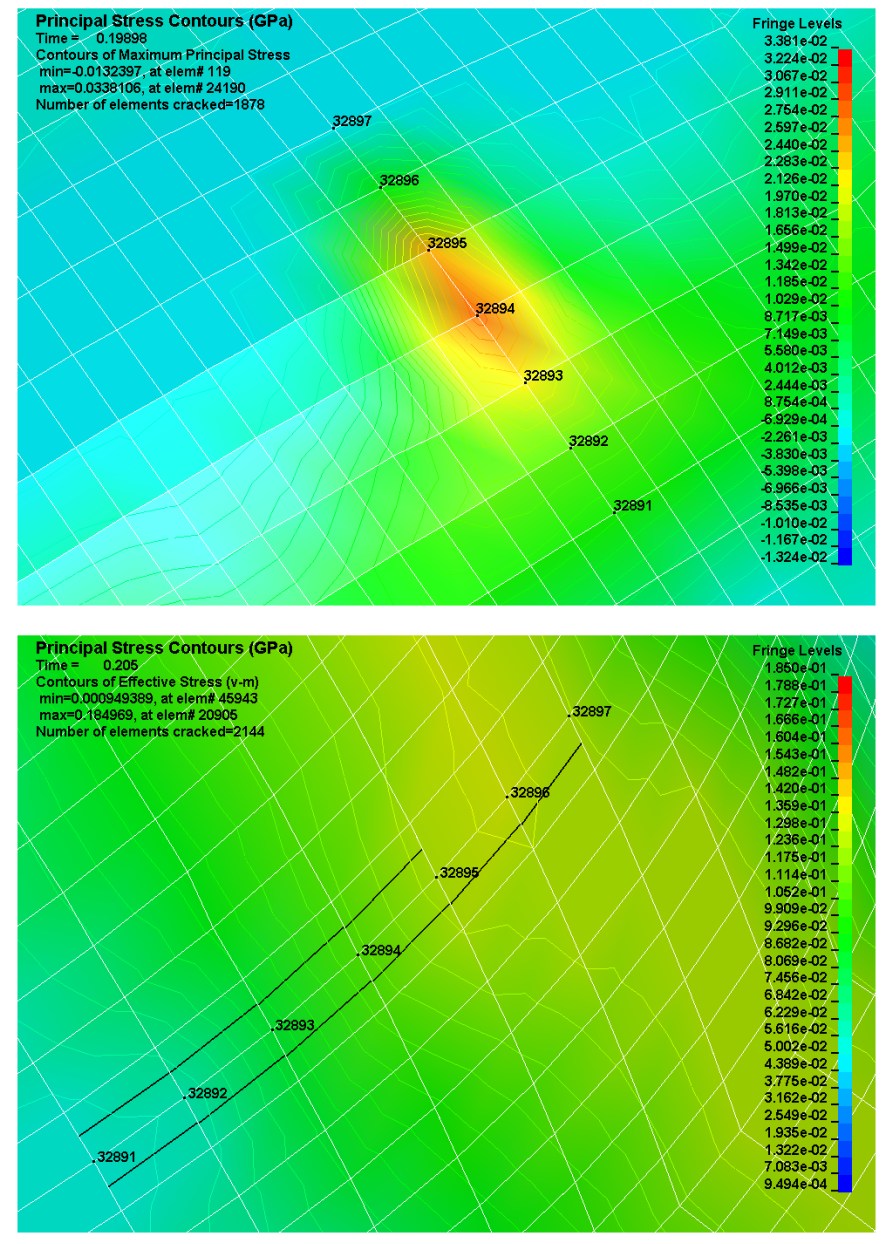

Figure 5. (a) Time just prior to crack appearance in the location $t=0.199 \mathrm{~ms}$ and (b) $\mathrm{x}, \mathrm{y}, \mathrm{x}$ locations of the nodes near the crack formed and propagated in the location $t=0.205 \mathrm{~ms}$.

the terminal crack velocity $(\mathrm{m} / \mathrm{s})$ values[14] for selected glasses are: soda lime glass $=1460-1600$, Mirror glass $=1520$, Borosilicate crown glass $($ BK-7) $=1677-1800$, and Fused silica $=2100$ 2500. In addition, from figure 5(b), the advancement of the formed cracks from the moment they are initiated (node 32894 at time 0.19898 ) to the position of the advancing crack tip (node 32891 or node 32897 , crack propagating in both directions from the source) yields a crack velocity value of $\sim 2000 \mathrm{~m} / \mathrm{sec}$ (based on the distance travelled $\sim 12 \mathrm{~mm}$ and the time $\Delta t=0.005 \mathrm{~ms}$ ), which is close to the terminal velocity of the Borosilicate (BK-7) glass reported in the literature. This agreement is attributed to our preliminary FEM model, which does not include water surrounding the PMT bulb and its interaction with the glass. This model is successful in predicting initial crack velocity (close to the terminal velocity) when the cracks initiate. As the cracks propagate, the fracture energy is spent and the crack velocity decreases. This aspect is not included in the current model. Our FEM model in its present form cannot capture the entire cracking dynamics.

The discrepancy in the velocity values was not surprising, because of the circumstances of the PMT failure induced in the prototypic testing. Being completely submerged in water and highly pressurized, we hypothesized that the crack velocities would measure lower than typical accepted 
values, as some of the fracture energy would be transferred to the water. Our approach to crack velocity measurement is unique in that we are able to observe the crack propagation in situ and measure as it progresses in real time.

The numerical predictions on (a) the location of PMT failure initiation and (b) the hydrostatic pressure level at failure initiation agree within errors of with the experimental results deduced from the BNL hydrostatic failure PMT tests. Specifically, the instability or buckling analysis as well as the glass cracking model simulations reveal that failure can be initiated at hydrostatic pressures as low as 294 psi and that the failure of the PMT is initiated at its cylindrical base. Repeated hydrostatic failure experiments at BNL have confirmed both the location of instability and the hydrostatic pressure threshold. The crack velocities measured by our method showed higher values at the middle section of the PMT, as predicted by the FEM model.

\section{Conclusion}

Our results show crack velocities in photomultiplier glass bulbs measured using the high-speed video method are well below reported terminal crack propagation velocities. Some of the limitations of our experimental measurement include video resolution, frame rate, and lighting of the sample, which affect our ability to accurately identify the crack tip at its initiation. We have made reasonable accommodation for these in our estimate of systematic errors. This method of observation gives us important information about the nature of the failure of PMT bulbs under hydrostatic pressure. With additional technical investment, this method could provide precise crack propagation data for in situ PMT bulb fractures. Nevertheless the data from these tests has been compared to a detailed numerical evaluation of crack formation. These are difficult simulations requiring input of material properties. They show that our data on the localization the cracks at the middle section of the PMT, as well as the pressure at failure can be reproduced with the FEM model.

\section{Acknowledgments}

The authors acknowledge support from BNL. SKS acknowledges the support from the Kyocera Corporation in the form of Inamori Professorship.

\section{References}

[1] S. Fukuda et al., The Super-Kamiokande detector, Nucl. Instrum. Meth. A 501 (2003) 418.

[2] J. Boger et al., The Sudbury Neutrino Observatory, Nucl. Instrum. Meth. A 449 (2000) 172.

[3] V. Barger et al., in Report of the US Long baseline neutrino experiment study, arXiv: 0705.4396 [FERMILAB-0801-AD-E] [BNL-77973-2007-IR].

[4] J. Goon et al., The long-baseline neutrino experiment conceptual design report - The LBNE water Cherenkov detector, April 10 (2012).

[5] K. Abe et al., Letter of intent: the Hyper-Kamiokande experiment detector design and physics potential, arXiv:1109.3262.

[6] M. Diwan et al., Underwater implosions of large format photo-multiplier tubes, Nucl. Instrum. Meth. A 670 (2012) 61. 
[7] J. Ling et al., Implosion chain reaction mitigation in underwater assemblies of photomultiplier tubes, Nucl. Instrum. Meth. A 729 (2013) 491.

[8] M. Diwan et al., Future long-baseline neutrino facilities and detectors, Adv. High Energy Phys. (2013) 460123.

[9] LS-DYNA, Version 9.71, Livermore Software Technology Corporation (LSTC), Livermore U.S.A.

[10] L. Schwer, The Winfrith concrete model: beauty or beat? Insights in the Winfrith concrete model, talk given at the $8^{\text {th }}$ European LS-DYNA users conference, May 23-24, Strasburg, France (2011).

[11] Y.D. Murray, Users manual for LS-DYNA concrete material model 159, Publication No. FHWA-HRT-05-062, US Department of Transportation, McLean, U.S.A. (May 2007).

[12] Truegrid Software, XYZ Scientific Applications, Livermore, U.S.A.

[13] photomultiplier tube R7081-02 data sheet, available at http://www.hamamatsu.com/.

[14] G. Quinn, Fractography of ceramics and glasses, National Institute of Standards and Technology, U.S.A. (2007). 